\title{
$1 \quad$ Numerical performance analysis of acoustic Doppler 2 velocity profilers in the wake of an axial-flow marine hydrokinetic turbine
}

\author{
Marshall Richmond*, Samuel Harding, Pedro Romero-Gomez \\ Hydrology Group, Pacific Northwest National Laboratory, Richland, WA, 99352
}

\begin{abstract}
6 Abstract
7 The use of acoustic Doppler current profilers (ADCPs) for the characterization of flow conditions in the vicinity of both experimental and full scale marine hydrokinetic (MHK) turbines is becoming increasingly prevalent. The computation of a three dimensional velocity measurement from divergent acoustic beams requires the assumption that the flow conditions are homogeneous among all beams at a particular axial distance from the instrument.

In the near wake of MHK devices, the mean fluid motion is observed to be highly spatially variable as a result of torque generation and energy extraction. We examine the performance of ADCP measurements through modeling of a virtual ADCP (VADCP) instrument in the wake of an MHK turbine resolved using unsteady computational fluid dynamics (CFD). This is achieved by sampling the CFD velocity field at equivalent locations to the sample bins of an ADCP, and performing the coordinate transformation from beam coordinates to instrument coordinates, and finally to global coordinates.

The stream-wise velocity deficit and tangential swirl velocity caused by the moving rotor lead to significant misrepresentation of the true flow velocity profiles by the VADCP. The most significant errors were observed in the transverse (cross-flow) velocity direction, with a relative error of $17 \%$ of the incident velocity. The relative RMS error over the rotor plane was calculate to be less than $2 \%$ of the incident velocity by a distance of $x / D \geq 2$, $x / D \geq 1$ and $x / D \geq 6$ for the stream-wise, transverse and vertical directions, respectively. For the default instrument orientation, A maximum error in the $V$-velocity occurs when the instrument is located directly behind the turbine, and a maximum $W$-velocity error is found at a lateral offset $y / D=0.20$. For an instrument directly behind the MHK device, the
\end{abstract}

\footnotetext{
* Corresponding author

Email address: marshall.richmond@pnnl.gov (Marshall Richmond)
} 
maximum error in the $U$-velocity and $V$-velocity occurs at a yaw angle of $\phi \approx 30^{\circ}$ and $\phi \approx 60^{\circ}$.

Keywords: Acoustic Doppler Current Profiler (ADCP), Non-homogeneous Velocity, Marine Hydro-kinetic Turbines

\section{Introduction}

The characterization of the flow conditions in the vicinity of marine hydrokinetic (MHK) turbines is of importance from the design phase through to the performance monitoring of the operational system. The extraction of energy from such dynamic flow environments is often associated with large changes in the local flow velocity in the vicinity of the device. Such spatial dependence of the flow requires the measurement to be accurate with a relatively high spatial and temporal resolution.

Acoustic Doppler current profilers (ADCPs) are often used in situations where simultaneous velocity measurements are desired throughout the water column. This is achieved through the simultaneous sampling of the water column using typically three to five diverging acoustic beams. The ability to configure the acoustic instrument for a wide range of applications has resulted in the broad use of ADCPs in oceans, rivers and estuaries. Other key advantages of ADCPs include the relative ease of deployment and limited post-processing requirements.

The operating principle of an ADCP requires the inherent assumption of homogeneity in the flow within the sampled area $[1,2]$. Each transducer emits an acoustic signal from the device and processes the reflected signal returned from a range of distances through the water column. The frequency shift from the transmitted acoustic signal is used to calculate the velocity in the direction of the acoustic beam [1]. To calculate a three-dimensional velocity vector, the velocities in at least three non-parallel beam directions must be measured. In order to measure a number of beam directions from a single device, the beam directions are divergent.

The reflected acoustic signal is range-gated by the instrument to allow the flow velocity to be sampled at a number of locations throughout the water column $[3,4]$. The location corresponding to each gated return signal is referred to as a sample bin. The length of the acoustic beam signal used to calculate the velocity at each sample bin is referred to as the bin length. The flow nearest to the instrument is unable to be measured due a minimum time delay required between each transducer transmitting the acoustic pulse and receiving the reflected signal [4]. The distance to the first sample bin is 

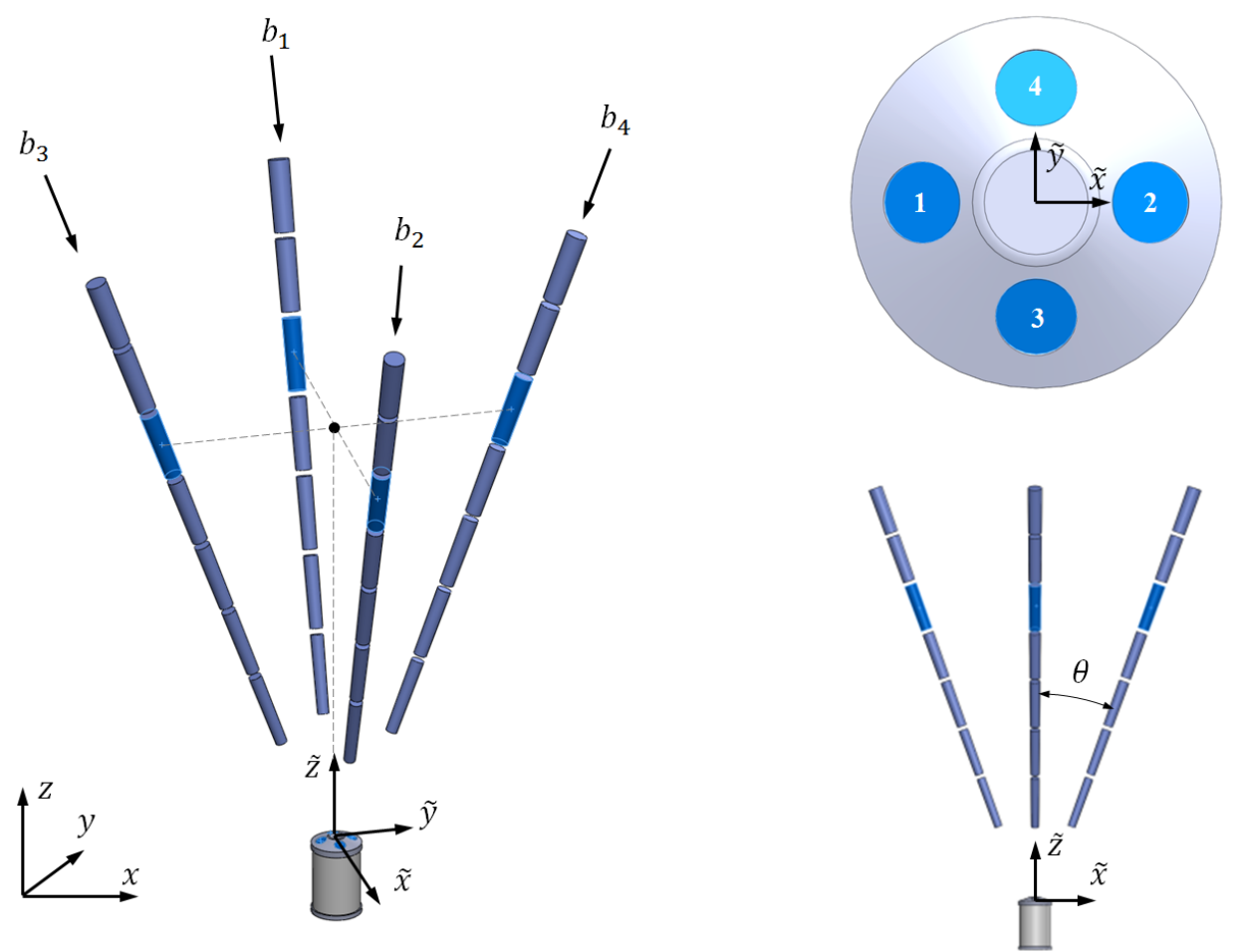

Figure 1: Global, instrument, and beam coordinate system notation. The location of bin 5 is highlighted in blue in the left hand figure, and the location of the reference velocity is shown with a black dot. The origin of the global coordinate system is shown in Figure 2. The beam numbering is shown in the $x-y$ plane in the right hand figure.

called the blanking distance. The rate of velocity measurements output by the instrument is referred to as the sampling frequency.

The beam-wise velocities from corresponding bin locations are transformed to calculate a three-dimensional velocity component, notionally located at the same axial distance from the instrument along its central axis, as shown in Figure 1. This process, described in detail in Section 4.1, assumes that the velocity is identical in all the bin locations in a plane perpendicular to the central axis of the instrument. Due to the divergent beam directions, the horizontal distance between bins increases with the distance from the instrument.

While the applicability of ADCPs to measure mean incident flow velocities is well established, the performance of the device in unsteady flows requires a more detailed analysis. This work predominantly involves the comparison of ADCP results with a reference velocimeter or simulation. 
The primary challenge in verifying the performance of ADCPs in the field is the deployment of a suitable sensor to quantify such a reference velocity.

A number of studies have compared ADCP measurements with acoustic Doppler velocimeters (ADVs) which measure the velocity at a single point $[5,6,7]$. Of particular relevance to this study, Neary et al. compare the mean velocity measured by a diverging-beam ADCP and an ADV in the wake of an experimental scale horizontal-axis turbine [7]. This comparison highlighted that the non-homogeneous flow of the near wake can lead to significant measurement errors when the homogeneity assumption of a diverging beam instrument is not valid [8]. Nystrom et al. conclude that quantities such as turbulent kinetic energy (TKE), turbulent intensity (TI) and Reynolds stresses can be determined with the ADCPs only after introducing further assumptions to simplify the non-homogeneity of turbulence [9].

A virtual ADCP (VADCP) can be modeled to represent the sampling geometry of an ADCP in a representative velocity field. This velocity field can be conveniently generated using computational fluid dynamics (CFD) to resolve three-dimensional flow effects at a relatively high spatial and temporal resolution.

The use of VADCPs to explore the sampling geometry effects is limited in existing literature. Boldt presents the error between the output of a virtual ADCP and a number of non-homogenous velocity field cases [10]. These include a range of roller eddy structures and turbulent field measurements. The velocity and Reynolds stresses calculated by a VADCP velocity measurements is compared with that calculated from the equivalent locations in a CFD velocity field, using a large eddy simulation (LES) by Tokyay et al. [11]. The modelling of a VADCP is primarily concerned with the effect of the vector transformation from individual beam-wise velocities to a three-dimensional velocity vector. The issue of Doppler noise [12] and other practical operational considerations are ignored.

\section{Aims and Objectives}

The numerical simulation presented herein is designed to examine how the flow field in the wake of an axial-flow MHK device risks being misrepresented by the velocity measurements of a diverging-beam ADCP instrument. A range of instrument locations and orientations are explored to quantify the deviation from the reference velocity as a result of the vector transformation algorithm applied to the diverging acoustic beam geometry.

By quantifying the theoretical error caused by the ADCP processing algorithm (in the absence of compounding practical and experimental error 
sources), this analysis aims to inform the appropriate deployment proximity and orientation for a range of accuracy requirements and particular flow interests.

\section{Simulation of MHK Wake Characteristics}

\subsection{Introduction to MHK Wake Effects}

The primary sources of non-homogeneity in the time-averaged velocity field in the wake of an MHK device can be considered as the superposition of two alterations to the mean flow field:

1. The extraction of energy from the flow by the turbine thrust and support structure drag creates a region of reduced stream-wise velocity behind the device. This velocity deficit is observed in the region known as the 'far wake', and is recovered through turbulent mixing with the downstream flow [13].

2. The generation of torque by the operational MHK imparts a reaction 'swirl' in the flow close to the rotor plane. This tangential velocity component is a characteristic of the 'near wake' and is dissipated by viscous shear forces [13].

A number of theoretical and experimental studies have been conducted to increase the understanding of wake behavior. A tabular summary of a number of these is compiled by Tedds et al. [14]. Data describing information of the behavior of the swirl is limited for the MHK application, even at experimental scale. However studies of this wake characteristic for the application of horizontal axis wind turbines suggest that the wake swirl is dissipated within five diameters downstream of the rotor plane [13].

\subsection{Device Geometry}

The MHK turbine used in this study is a full scale model of a threebladed, horizontal axis device on a monopole foundation. The key dimensions of the device and the global coordinate system used throughout the analysis are shown in Figure 2. This turbine was designed using blade element momentum (BEM) theory, and the profiles of the lifting surfaces were determined using the subsonic airfoil design tool 'XFOIL' [15]. The development of this device has been a multi-institutional effort involving design at Sandia National Laboratories [16], blade element momentum modelling at UC Davis [15], laboratory testing at Pennsylvania State University [17] and 



Figure 2: Coordinate system and geometry of MHK turbine. Dimensions are in meters.

hydraulic and biological performance assessments at the Pacific Northwest National Laboratory $[18,19]$.

The direction of rotation is in the counter-clockwise direction and the tip speed ratio $(T S R=)$ for the simulation was 4 . At this operating condition the thrust and power coefficient are equal to $C_{T}=0.79$ and $C_{P}=0.46$. This operating point represents the peak of the performance curve for the turbine power output.

\subsection{Velocity Notation and Coordinate Systems}

The coordinate systems and notation used throughout the VADCP analysis is presented below. While other beam numbering conventions exist, the conventions defined in this section are consistent with the VADCP results described and discussed herein.

The beam-wise velocity directions are defined as positive in the direction towards the transducers as shown in Figure 1. The instantaneous beam-wise velocity of beam $i$ is denoted $b_{i}$. The two opposing beam pairs are Beam 1 and Beam 2, and Beam 3 and Beam 4.

A Cartesian coordinate system is defined relative to the instrument with directions $[\tilde{x}, \tilde{y}, \tilde{z}]$. This is referred to as the instrument coordinate system. In the following analysis, Beam 2 is aligned in the direction of $\tilde{x}$ and Beam 4 with the $\tilde{y}$ axis. The instantaneous velocity vector aligned with instrument coordinate system is denoted $[\tilde{u}, \tilde{v}, \tilde{w}]$. 
The global coordinate system, $[x, y, z]$, is defined relative to the computational domain. This is conveniently aligned with the axes of the MHK device. The global coordinate system directions and origin are defined in Figure 2. The instantaneous velocity vectors in this reference system are denoted $[u, v, w]$.

The instantaneous velocity calculated by the vector transformation from beam-wise velocity vectors to global coordinates are denoted $\left[u_{c}, v_{c}, w_{c}\right]$. For comparison, the instantaneous reference velocity at the same axial distance from the device, at a point along the central axis of the instrument, are denoted as $\left[u_{r}, v_{r}, w_{r}\right]$.

The time-averaged velocity vectors are denoted using the upper-case notation of the equivalent instantaneous velocity vectors. This mean velocity is calculated over the entire CFD run duration of $180 \mathrm{~s}$, unless otherwise stated.

The incident flow speed at the turbine hub height is $U_{0}=2.0 \mathrm{~m} / \mathrm{s}$. The orientation of the beam directions and instrument coordinate systems are shown within the global coordinate system in Figure 1.

\subsection{Computational Fluid Dynamics Solution}

The flow in the wake of the MHK turbine was resolved using an unsteady CFD simulation of the full-scale device that was previously reported by Romero-Gomez and Richmond[24]. A detached eddy simulation (DES) with a $\kappa-\omega$ turbulence solver was implemented using the commercial software STAR-CCM+ v8 [20] to describe the turbulent flow. The computational domain of the $C F D$ was $16 D \times 8 D \times 4 D$ (stream-wise, transverse, vertical flow directions, respectively), where $D$ is the diameter of the swept rotor area. A hexahedral mesh was used with a total of $10.2 \mathrm{M}$ cells. Approximately $30 \%$ of these were located in the refined mesh in the vicinity of the rotor where increased spatial resolution was required for accurate resolution of the flow behavior about the lifting surfaces. A cylindrical region of the domain encompassing the rotor was rotated at $3.2 \mathrm{rad} / \mathrm{s}$ to simulate the turbine in operation with a tip speed ratio of 4.0 .

Field measurements of turbulence were previously examined at an energetic tidal site, and the turbulent intensity and length scales used in the present study are derived from that previous work [21]. The inflow velocity conditions followed a power law with a nominal velocity of $U_{0}=2.0 \mathrm{~m} / \mathrm{s}$, and contained representative turbulence characteristics of energetic tidal flows with the turbulence intensity of $T I=11 \%$. The synthetic-eddy-method presented by Jarrin et al. was used as a well-documented, widely used and computationally efficient method of generating coherent turbulent eddies in 


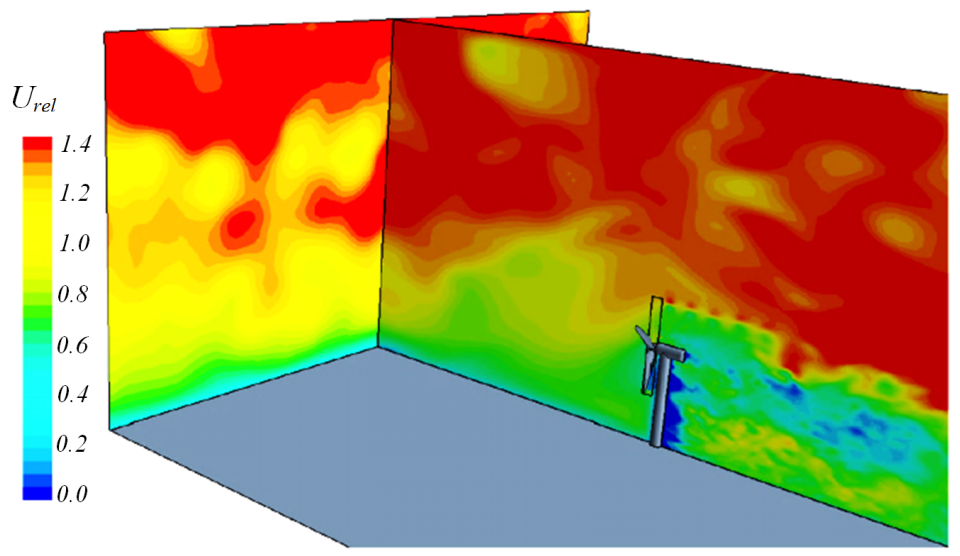

Figure 3: Instantaneous velocity field upstream of the MHK device and in the near wake, normalized by mean incident velocity of $2.0 \mathrm{~m} / \mathrm{s}$ [24]

the incident flow [22]. The Lagrangian integral time scale $\left(t_{\text {lag }}\right)$ was reported to equal $1.4 \mathrm{~s}$. The length scale resulted from multiplying $t_{\text {lag }} \times U_{0}=2.8 \mathrm{~m}$. This corresponds to the size of the most energetic eddies in the flow. The boundary conditions of the top and side surfaces were modeled as symmetry planes, with no flow recirculation at the outlet plane. The seabed and turbine structure were modeled as no-slip solid walls. Further details on the CFD modeling methods used are reported by Richmond and Romero-Gomez $[23,24]$. The resulting incident and near wake flow velocities, normalized by the nominal flow speed of $2.0 \mathrm{~m} / \mathrm{s}$ are presented in Figure 3 .

\subsection{Run Duration}

The CFD simulation was run for an initial warm-up period of $180 \mathrm{~s}$ and then the velocity at the locations corresponding to the bin centers of the VADCP were recorded for an acquisition period of $180 \mathrm{~s}$. The mean velocity components were shown to reach statistically stable values over this acquisition period.

\subsection{Velocity Field Characteristics}

The velocity field was sampled in vertical planes at $x / D=[1,2,3,4,6,10,12]$ downstream of the rotor plane. The relative magnitude of the wake effects can be considered by normalizing the flow field by $U_{0}$. Recalling the coordinate system defined in Figure 2, selected components of the mean velocity flow field in the $x-z$ plane are shown in Figure 4. 

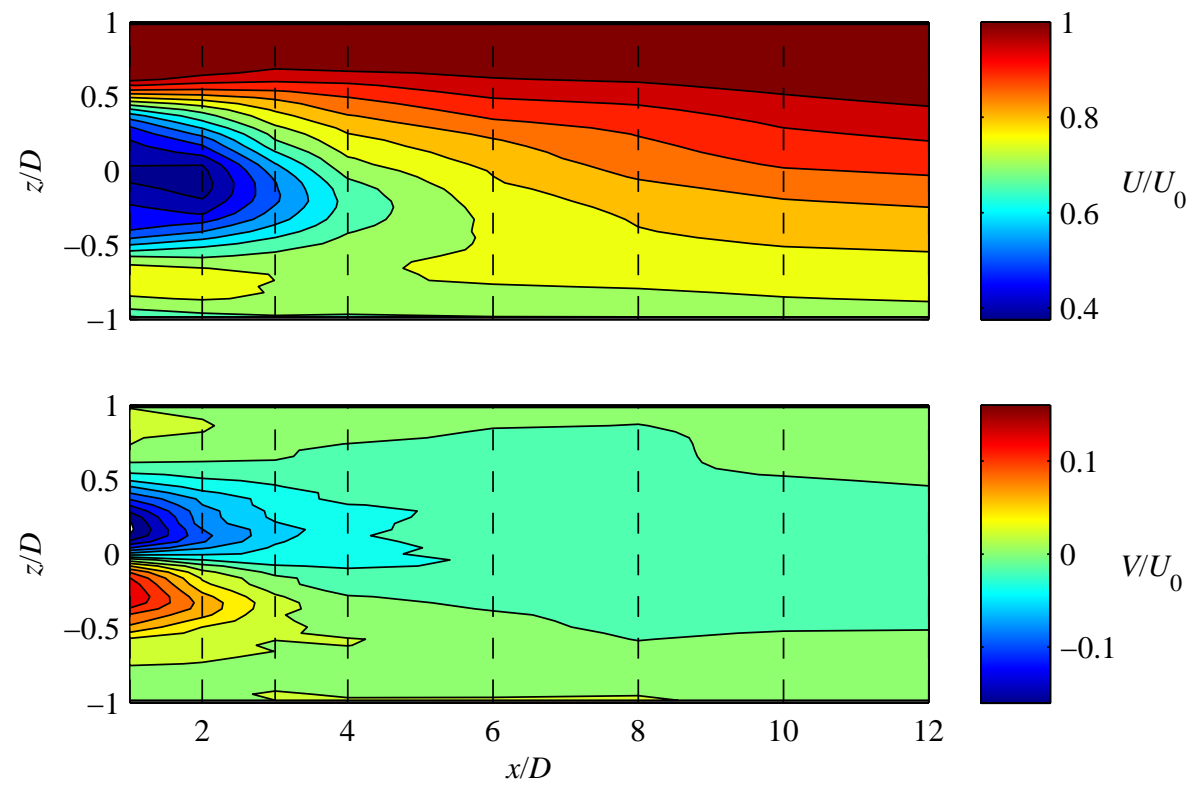

Figure 4: Normalized time-averaged velocity fields in the $x-z$ plane, showing recovery of stream-wise velocity deficit in the wake (top) and transverse component of wake swirl (bottom). The locations of the sample planes are indicated by dashed lines.

The qualitative results demonstrate the characteristic flow phenomena of an MHK turbine wake. In the top plot, a clear region of low streamwise velocity is observed directly behind the turbine rotor plane with a minimum velocity close to the rotor axis. The wake recovery is observed by the downstream flow mixing to a shear layer with the minimum flow velocity nearest the bottom of the computational domain. The stream-wise velocity deficit in the wake of the rotor is not symmetrical about the rotor axis in the $x-z$ plane. This was observed by Myers and Bahaj [13] in the experimental modeling of a $1 / 20$ th scale MHK device and is attributed to the additional velocity deficit caused by the supporting structure between the turbine nacelle and the seabed.

The bottom plot demonstrates the flow swirl as a result of the rotating blades about the $y$-axis. Tangential velocity components are evident in the transverse direction in the $x-z$ plane as the flow in the near wake rotates about the axis of the turbine rotor. This effect is dissipated more rapidly than the stream-wise velocity deficit, with significantly reduced evidence of swirl at $x / D \geq 5$. 


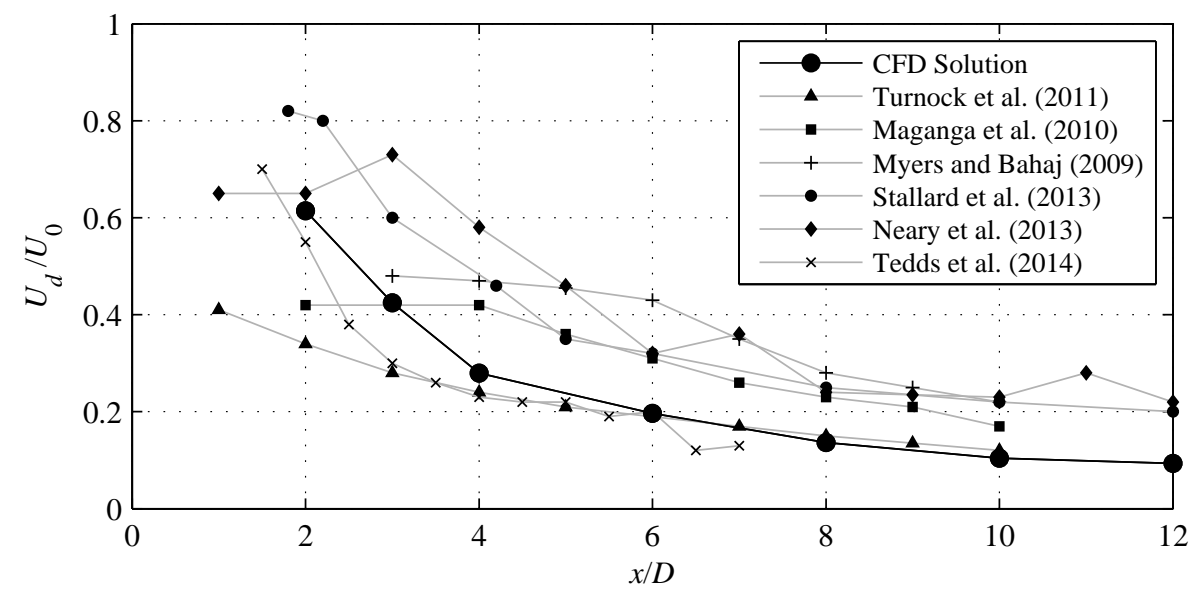

Figure 5: Decay of velocity deficit with distance downstream of rotor plane.

\subsection{Validation of CFD Results}

The velocity deficit in the stream-wise velocity, $U_{d}$, is calculated by Equation 1.

$$
U_{d}=U-U_{0}
$$

The wake recovery of the CFD velocity field was compared with published results from experimental and computational wake studies of threebladed MHK turbines in Figure 5.

The wake recovery behaves similarly to that behind horizontal axis wind turbines, where self-similarity exists in the wake [25]. As such, the rate of decay of the stream-wise velocity deficit for each set of experimental results approximates a power law defined by Equation 2 .

$$
\frac{U_{d}}{U_{0}} \approx a(x / D)^{-b}
$$

The wake recovery rate, $b$, was calculated by fitting a power law model to published results in the range $2<x / D<12$. The key experimental details and associated wake recovery rates are summarized in Table 1 . Direct comparison between each experimental setup is difficult due to the range of experimental methods, geometries, flow conditions, and turbine performance characteristics such as the thrust coefficient, $C_{T}$. Yet there are some aspects of the wake behavior that are consistent over all results. A monotonic decrease in velocity deficit is observed in all experiments as well as the 


\begin{tabular}{cccccccccc}
\hline Ref. & Method & $\begin{array}{c}D \\
\mathrm{~m}\end{array}$ & $\begin{array}{c}U \\
\mathrm{~m} / \mathrm{s}\end{array}$ & $\begin{array}{c}R e_{D} \\
-\end{array}$ & $\begin{array}{c}T I \\
\%\end{array}$ & $\begin{array}{c}T S R \\
-\end{array}$ & $\begin{array}{c}C_{T} \\
-\end{array}$ & $\begin{array}{c}C_{P} \\
-\end{array}$ & $\begin{array}{c}b \\
-\end{array}$ \\
\hline$[26]$ & CFD & 20 & 2 & $4.0 \times 10^{7}$ & - & 9.2 & 0.69 & - & 0.54 \\
{$[27]$} & Exp. & 0.7 & 0.8 & $5.6 \times 10^{5}$ & 8 & - & - & - & 0.57 \\
{$[13]$} & Exp. & 0.8 & 0.8 & $6.4 \times 10^{5}$ & 6 & - & 0.77 & - & 0.68 \\
{$[28]$} & Exp. & 0.27 & 0.45 & $1.2 \times 10^{5}$ & 10 & 4.7 & 0.82 & - & 0.81 \\
{$[7]$} & Exp. & 0.5 & 0.4 & $2.0 \times 10^{5}$ & 9.8 & 5.8 & 0.52 & 0.48 & 0.92 \\
{$[14]$} & Exp. & 0.5 & 0.89 & $4.5 \times 10^{5}$ & 2 & 6.15 & 1 & 0.34 & 1.04 \\
- & CFD & $\mathbf{4 . 8 8}$ & $\mathbf{2}$ & $\mathbf{9 . 8} \times \mathbf{1 0}^{\mathbf{6}}$ & $\mathbf{1 1}$ & $\mathbf{4}$ & $\mathbf{0 . 7 9}$ & $\mathbf{0 . 4 6}$ & $\mathbf{1 . 0 8}$ \\
\hline
\end{tabular}

Table 1: Decay of normalized stream-wise velocity deficit with distance downstream of rotor plane, in order of increasing wake recovery rate. The details of the CFD set-up used in the present study is highlighted in bold.

CFD results. Also, the wake is never observed to have fully recovered by $x / D=12$.

The theoretical exponent of the wake recovery for the centreline streamwise velocity of self-similar axi-symmetric wake is $b=0.66$ [25]. The wake recovery rate of the CFD results used in this analysis $(b=1.08)$ was near the maximum of the range of recovery rates observed in literature $(0.54 \leq b \leq 1.04)$. This means that the velocity deficit in the simulated wake of the present study dissipates more rapidly than the majority of other studies. In turn, it can be assumed that the VADCP error values calculated in this analysis are conservative estimates, and would be larger in the more persistent wakes cited in the other studies.

The available quantitative results regarding the expected reduction of the swirl in the near wake is limited. An experimental study of a threebladed MHK device at experimental scale by Tedds et al. [14] measured a 40-50\% reduction in tangential velocity components between $x / D=1$ and $x / D=7$. However the turbulence intensity of the flow in these reduced scale experiments was $\mathrm{TI}=2 \%$ and so mixing in the wake region can be expected to be significantly less than that in the case modeled in this analysis $(\mathrm{TI}=11 \%)$. Studies of swirl in the near wake of horizontal axis wind turbines suggest that the tangential velocity component is dissipated within five diameters downstream of the rotor plane [13]. The tangential velocity component was observed to reduce by approximately $75 \%$ in the CFD results used in this analysis, between the stream-wise locations of $x / D=1$ and $x / D=4$. Again this represents a conservative estimate of the swirl velocity, as the tangential component of the near wake velocity is dissipated at least 
as rapidly as expected.

In addition, the predicted power and thrust coefficients from the CFD model were compared to BEM and laboratory results for these performance coefficients $[24,17]$. The results were found to be mostly in agreement in both cases supporting the assumption that the CFD calculation successfully represented realistic flow conditions for use in this analysis. Differences were likely due to the laboratory measurements being done on a reduced scale model in a water tunnel as compared to the CFD simulation for a full-scale turbine in a open domain.

\section{Virtual Acoustic Doppler Current Profiler model}

\subsection{VADCP Theory}

The virtual ADCP instrument is modeled by extracting velocity values from the CFD model at locations which correspond to the center of each bin of the four diverging acoustic beams. The velocities at equivalent bin heights directly above the instrument are also recorded as this is the notional position of the velocity measurement. The velocity processing of the VADCP is calculated in the following steps:

1. The location, blanking distance, bin length, orientation, and sample frequency of the virtual instruments are specified. In the analysis presented herein, the VADCP geometry represents a 4-beam ADCP configuration. The blanking distance is $0.75 \mathrm{~m}$, the bin length is 0.50 $\mathrm{m}$ and sample frequency of the instrument is $2 \mathrm{~Hz}$. The influence of instrument location and orientation is presented in sensitivity analyses in Section 5.

2. The velocity vectors are extracted from the unsteady CFD solution at the locations of each bin center of the four diverging acoustic beams. The velocity vectors are also extracted for a vertical acoustic beam with bins at the same axial distance from the instrument, to be used as a reference velocity.

3. The velocity vectors are converted from Cartesian coordinates into beam-wise velocity vectors. Letting the velocity vector of beam $i$ at bin $j$ be defined as $a_{i, j}=\left[u_{i, j}, v_{i, j}, w_{i, j}\right]$ and the unit beam direction vector be denoted as $\hat{d}_{i}=\left[\Delta x_{i}, \Delta y_{i}, \Delta z_{i}\right]$, then the component of the velocity vector in the direction of the acoustic beam is calculated by Equation 3.

$$
b_{i, j}=a_{i, j} \cdot \hat{d}_{i}
$$


4. The beam-wise velocity vectors are combined to calculate a threedimensional velocity vector in the instrument coordinate system. Defining the flow velocity in the direction of beam $i$ as $b_{i}$, with positive velocity being towards the instrument, the coordinate transformation from beam coordinates to instrument coordinates is given by Equation 4 $[11,3]$. The angle of divergence of each acoustic beam from vertical, which ranges from $20^{\circ}-25^{\circ}$ for most commercial ADCP units, is taken as $\theta=20^{\circ}$ in this analysis.

$$
\begin{aligned}
\tilde{u}_{i} & =\frac{b_{1}-b_{2}}{2 \sin \theta} \\
\tilde{v}_{i} & =\frac{b_{3}-b_{4}}{2 \sin \theta} \\
\tilde{w}_{i} & =-\frac{b_{1}+b_{2}+b_{3}+b_{4}}{4 \cos \theta}
\end{aligned}
$$

The coordinate transform from four beams to three Cartesian velocity components is an over-determined system, and so the mathematical transformation of Equation 4 is not a unique solution.

5. The velocity vector is transformed from the instrument coordinate system to the global coordinate system using the roll $(\psi)$, pitch $(\vartheta)$ and yaw $(\phi)$ angles specified in the ADCP orientation input matrix. The Euler angle matrix transformation is given in Equation 5.

$R=\left[\begin{array}{ccc}\cos \psi & -\sin \psi & 0 \\ \sin \psi & \cos \psi & 0 \\ 0 & 0 & 1\end{array}\right]\left[\begin{array}{ccc}\cos \vartheta & 0 & \sin \vartheta \\ 0 & 1 & 0 \\ -\sin \vartheta & 0 & \cos \vartheta\end{array}\right]\left[\begin{array}{ccc}1 & 0 & 0 \\ 0 & \cos \phi & -\sin \phi \\ 0 & -\sin \phi & \cos \phi\end{array}\right]$

The relationship between the velocity components in the instrument coordinate system and those in the global coordinate system is then given in Equation 6.

$$
\left[\begin{array}{c}
u_{i} \\
v_{i} \\
w_{i}
\end{array}\right]=\left[\begin{array}{c}
\tilde{u}_{i} \\
\tilde{v}_{i} \\
\tilde{w}_{i}
\end{array}\right] R
$$

6. The calculated velocity vectors are compared with the co-located CFD velocity vectors to perform an error analysis. The calculated velocity, $\left(u_{c}, v_{c}, w_{c}\right)$, is derived using the coordinate transform described in steps 2-5 and compared against the reference velocity at the equivalent axial 


\begin{tabular}{cccccccc}
\hline \multirow{2}{*}{$\begin{array}{c}\text { Lateral offset } \\
(y / D)\end{array}$} & \multicolumn{8}{c}{ Longitudinal distance from of rotor plane $(x / D)$} \\
\cline { 2 - 7 } & 1 & 2 & 3 & 4 & 6 & 8 & 10 \\
\hline 0 & $\checkmark$ & $\checkmark$ & $\checkmark$ & $\checkmark$ & $\checkmark$ & $\checkmark$ & $\checkmark$ \\
0.25 & $\checkmark$ & $\checkmark$ & $\checkmark$ & $\checkmark$ & & & \\
0.5 & $\checkmark$ & $\checkmark$ & & & & & \\
\hline
\end{tabular}

Table 2: Matrix of VADCP instrument locations

distance from the ADCP, $\left(u_{r}, v_{r}, w_{r}\right)$. For every sampled time step, both a calculated and a reference velocity vector are recorded.

7. The mean error as a function of the acquisition time, $t_{a}$, is defined for the streamwise direction in Equation 7. This equation is modified for the transverse and vertical velocity error by substituting $v$ and $w$ for the $u$ velocity components, respectively,

$$
U_{e}\left(t_{a}\right)=\frac{1}{t_{a}} \int_{0}^{t_{a}}\left(u_{r}(t)-u_{c}(t)\right) \mathrm{d} t
$$

Note that this process models the effect of using four separate beam location to infer a single velocity measurement. The effect of Doppler noise and other known issues associated with field deployments, such as accuracy of positioning and acoustic signal attenuation, have been neglected [21].

\subsection{Locations of VADCP instruments}

Virtual ADCP instruments were positioned at the combinations of longitudinal and transverse positions are indicated in Table 2.

Point probes are used to extract the unsteady velocity data at $16 \mathrm{~Hz}$ from the CFD simulation from the locations of the ADCP bins shown in Figure 6. This data set was down-sampled to $2 \mathrm{~Hz}$ as a representative sampling frequency of an ADCP instrument. Additional probes were added upstream of the MHK device to record simulated inflow conditions.

The error between the calculated and reference velocities of the downsampled $2 \mathrm{~Hz}$ data follows a Gaussian distribution over the solution period. The maximum standard deviation of the error velocities is observed in the VADCP instrument measurements closest to the turbine and decreases with wake recovery. The depth averaged standard deviations of the relative error velocities are calculated as $0.37-0.54 \mathrm{~m} / \mathrm{s}, 0.35-0.51 \mathrm{~m} / \mathrm{s}$, and $0.16-0.26 \mathrm{~m} / \mathrm{s}$ for the stream-wise, lateral and vertical flow directions, respectively. 


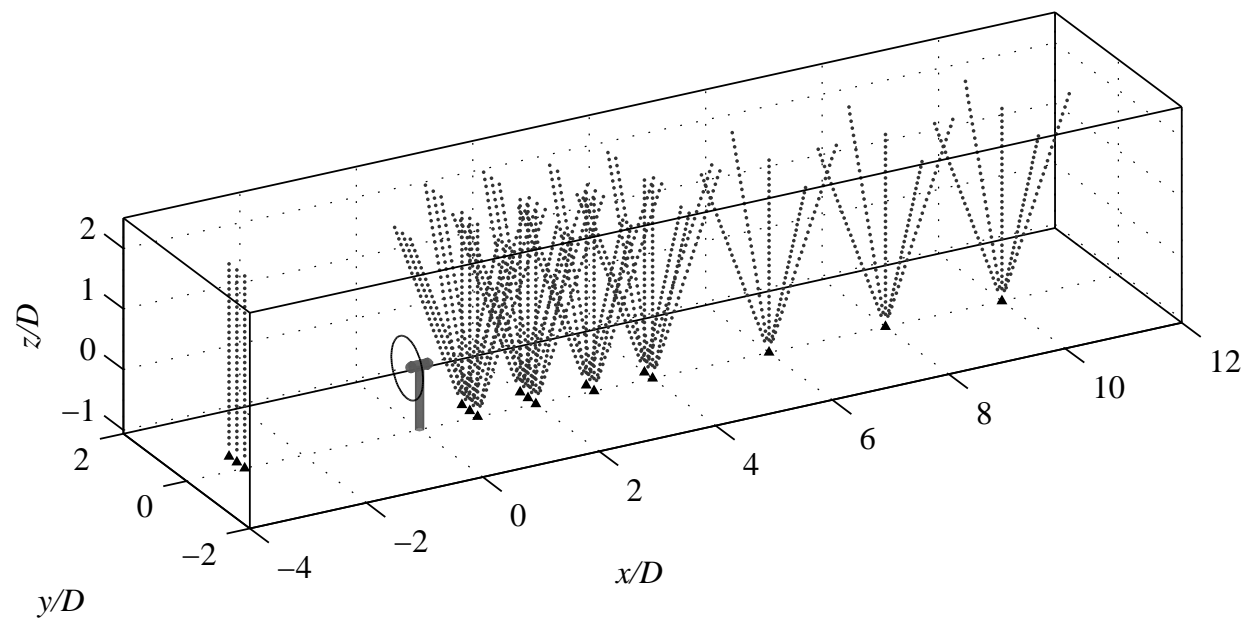

Figure 6: Schematic of probes for VADCP bin center locations. A sub-region of the computational domain is shown for clarity of detail.

\subsection{Scaling Effects}

The relative locations of the acoustic beams are expected to be similar when an ADCP is modeled in the wake of larger MHK devices. Assuming that the hub height and turbine diameter scale in an approximately linear manner, the ADCP beams will capture similar regions of the flow, as shown in Figure 7. The spatial resolution of the measurements increases for larger MHK devices, with more samples within the rotor plane.

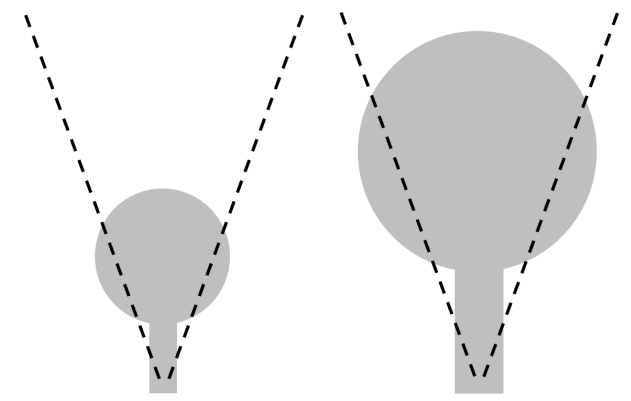

Figure 7: Schematic of ADCP acoustic beams (dashed lines) in the wake of linearly scaled MHK turbine (frontal area of swept blade diameter and monopile support shown in gray). 


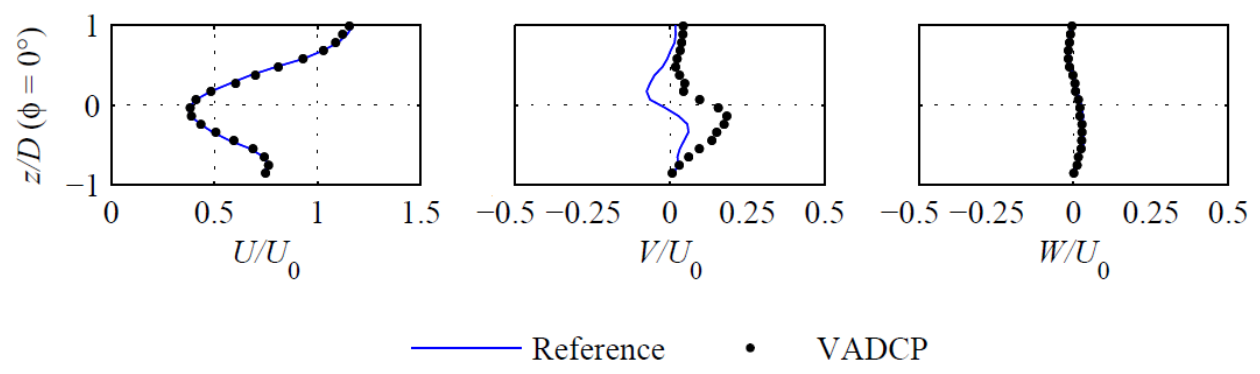

Figure 8: Comparison of calculated VADCP results and reference flow field velocities from CFD velocity field at $x / D=2, y / D=0$.

\section{CFD Flow Field Results}

The results of the calculated VADCP velocity measurements are compared with the reference velocity for the CFD solution for the turbine wake.

\subsection{Near wake results}

In the default instrument orientation, the stream-wise velocity deficit is qualitatively resolved by the VADCP at the position $(x / D, y / D)=(2,0)$, however the tangential velocity components introduced by swirl show significant errors, as shown in Figure 8. The maximum relative error magnitude in the transverse flow direction is $V_{e} / U_{0}=0.17$ near the turbine hub height.

This can be explained by the orientation of the VADCP being aligned with the flow direction. At this orientation, Beam 1 and Beam 2 are in the $x-z$ plane, and Beam 3 and Beam 4 are in the $y-z$ plane. Due to the nature of the wake swirl and the location of the instrument, the $V$-velocity components observed by Beam 3 and Beam 4 are equal at a particular vertical distance from the instrument. The error is the result of the $V$-velocity at the beam locations being different from that directly above the instrument where the reference velocity is taken. The reduction in the calculated $V$ velocity component above the turbine axis is due to the greater separation of the beams at this distance from the instrument. As such, the area of peak swirl in the upper portion of the rotor plane is not able to be captured.

When the VADCP is located at $y / D=0$, where the actual vertical component of the swirl velocity is zero. With Beam 1 and Beam 2 orientated in the $x-z$ plane at $y / D=0$, the beams are unable to detect any vertical swirl velocity. As discussed earlier, the beam velocities of Beam 3 and Beam 4 are of equal magnitude and opposite sign. In the numerator of Equation 4c, the 


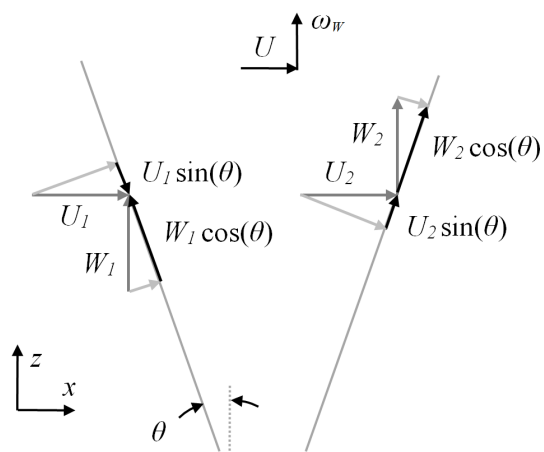

(a)

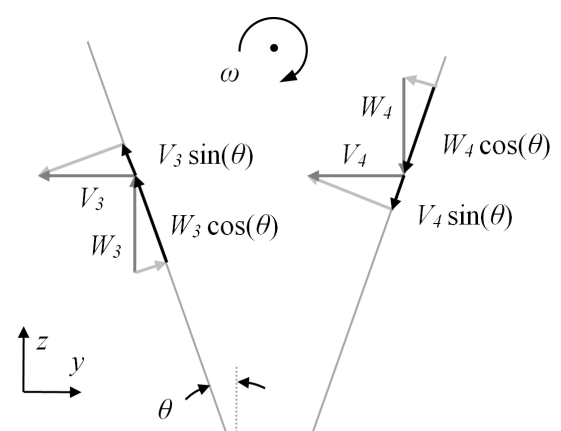

(b)

Figure 9: Schematic diagram of beam-wise velocity vectors of VADCP in rotating flow. In the default orientation, Beams 1 and 2 are able to detect the stream-wise flow velocity, $U$, as well as the vertical velocity component of the swirl velocity, $\omega_{W}$, when $y / D \neq 0$. Beams 3 and 4 detect both the vertical and transverse components of the swirl velocity.

beam velocities sum to zero. The vector diagram of this scenario is shown in Figure 9.

Due to the inherent difficulties in installing instruments in energetic flows, it is important to examine the cases where the ADCP is not directly downstream of the turbine. The transverse location of one half of the turbine radius is of particular interest as the swirled flow is clearly manifested in both the $V$-direction and $W$-direction at this location, as shown in Figure 10. However, the VADCP measurements of the analytical flow field show that neither is resolved correctly by the coordinate transform algorithm. The maximum relative error magnitude in these velocities is $V_{e} / U_{0}=0.12$ and $W_{e} / U_{0}=0.04$.

When the ADCP is one radius from the center-line of the nacelle $(y / D=$ $0.5)$, the swirl velocity is significantly reduced. However the calculated velocity incorrectly detects some transverse velocity component with a relative error magnitude of $V_{e} / U_{0}=0.10$ at the hub height.

\subsection{Far wake results}

The performance of the VADCP improves with downstream distance from the turbine, as the wake becomes more homogeneous with stream-wise velocity recovery and swirl dissipation. This is observed by the reduction in the RMS error of the normalized velocity components, shown in Figure 11.

With a blanking distance of $0.75 \mathrm{~m}$ and a bin length of $0.5 \mathrm{~m}$, bin numbers 5-14 are within the vertical range of the rotor plane. Recalling the error 

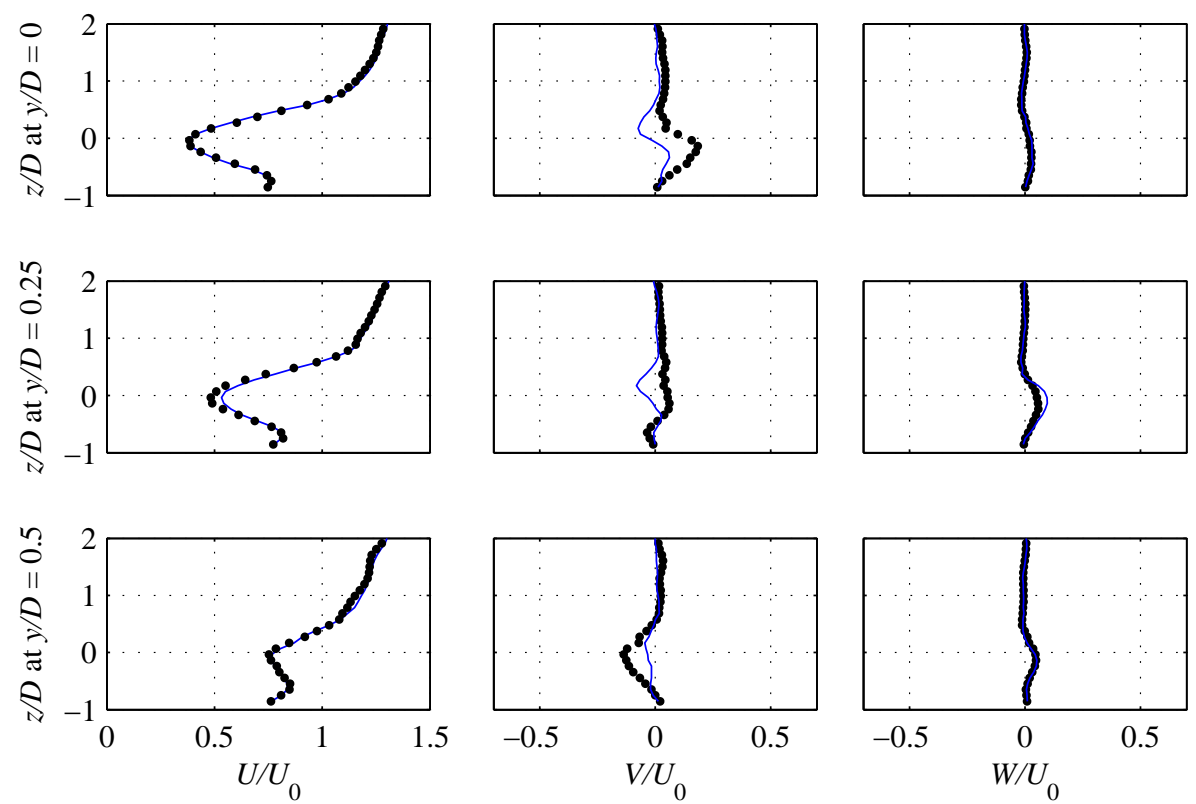

Reference

- $\quad$ VADCP

Figure 10: Comparison of calculated VADCP results and reference CFD flow field velocities for three transverse locations at $x / D=2$.

definition of Equation 7, the RMS error for the the stream-wise velocity over the rotor plane is calculated by Equation 8 .

$$
U_{e, \mathrm{RMS}}=\sqrt{\sum_{i=5}^{14}\left(U_{e, i}\right)^{2} / 10}
$$

Figure 11 also indicates the relative significance of the error in the transverse velocity component component for the case where the VADCP is directly behind the turbine in and aligned with the flow direction $(y / D=0$, $\left.\phi=0^{\circ}\right)$. The RMS velocity errors for both the stream-wise and vertical velocity directions are less than $2 \%$ of $U_{0}$ for $x / D \geq 2$. The RMS error of the normalized transverse velocity decreases approximately following a power law reduces to $2 \%$ at a location of $x / D \approx 6$. 


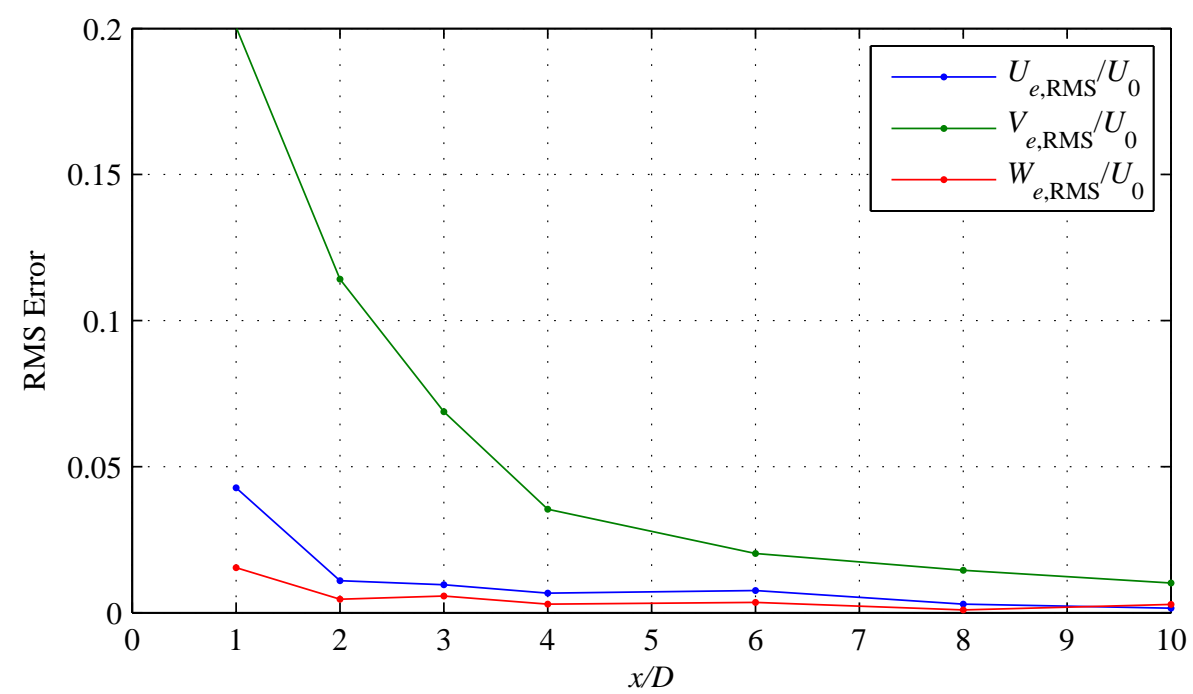

Figure 11: Mean RMS error of the normalized velocity components over the plane of the turbine rotor, as a function of distance from the rotor $\left(y / D=0, \phi=0^{\circ}\right)$.

\section{Analytical Flow Field Results}

A simplified representation of the mean flow velocities was implemented in MATLAB to examine the positional sensitivity of the VADCP measurements in the near wake of the MHK turbine in more detail. This flow field was used to approximate the time-averaged CFD simulation results by fitting to the CFD results at $x / D=2$.

The purpose of developing an analytical flow field solution is so that the mean velocities at all locations in the flow field are readily available and VADCP calculations can be done quickly. To achieve this flexibility using the CFD results becomes computationally expensive since the unsteady velocity field is sampled as the simulation progresses.

The undisturbed longitudinal velocity follows a power law profile. Using the self-similarity law, the velocity deficit in the wake of the turbine is approximated using an exponential profile with a maximum velocity reduction at the hub height [29]. The swirl magnitude is modeled using a Burgers vortex [30]. Both of these models have been used by $\mathrm{Hu}$ et al. [31] to model the wake of horizontal axis wind turbines.

The superposition of these wake effects is represented in the analytical flow field defined mathematically by Equation 9 . 

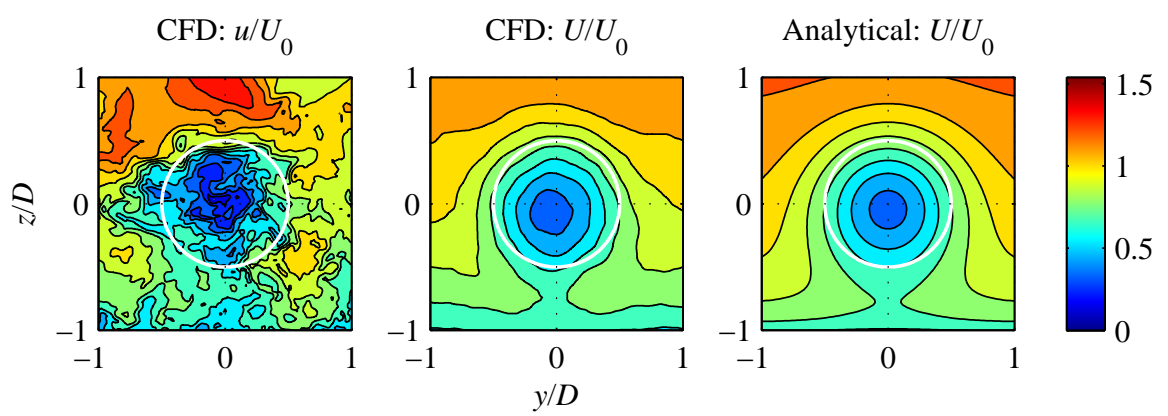

Figure 12: Development of analytical flow field solution from CFD velocities at $x / D=2$. The turbine rotor diameter is indicated with a white line.

\begin{tabular}{cccccc}
\hline $\begin{array}{c}A \\
\mathrm{~ms}^{-1}\end{array}$ & $\begin{array}{c}B \\
-\end{array}$ & $\begin{array}{c}C \\
\mathrm{~ms}^{-1}\end{array}$ & $\begin{array}{c}D \\
\mathrm{~m}\end{array}$ & $\begin{array}{c}E \\
\mathrm{~s}^{-1}\end{array}$ & $\begin{array}{c}F \\
\mathrm{~m}\end{array}$ \\
\hline 2.2 & 6 & 1.4 & 9.4 & 0.25 & 0.68 \\
\hline
\end{tabular}

Table 3: Optimized analytical flow field constants

$$
\begin{aligned}
U & =A\left(\frac{z+z_{T}}{z_{T}}\right)^{1 / B}-C e^{-16 r^{2} \ln (2) / D^{2}} \\
V & =-\frac{E F^{2}}{r}\left(1-e^{-r^{2} / F^{2}}\right)\left(\frac{z}{r}\right) \\
W & =\frac{E F^{2}}{r}\left(1-e^{-r^{2} / F^{2}}\right)\left(\frac{y}{r}\right)
\end{aligned}
$$

Here, $r$ is the radial distance from the turbine axis $\left(r=\sqrt{y^{2}+z^{2}}\right)$ and $z_{T}$ is the distance of the turbine rotor axis from the tower base. The constants in Equation 9 were determined using a multi-variate optimization using MATLAB to deduce the combination of values which most closely represented the mean CFD results in the range $-1<y / D<1$ and $-1<$ $z / D<1$. The values calculated from the optimization process are are summarized in Table 3. Contour plots showing the velocity deficit in the stream-wise velocity and the affect of swirl on the vertical velocity are shown in Figure 12.

A comparison between the analytical flow field solution and the CFD results at $x / D=2$ is presented in Figure 13. The velocity deficit in the stream-wise direction show good agreement at all lateral locations, while 

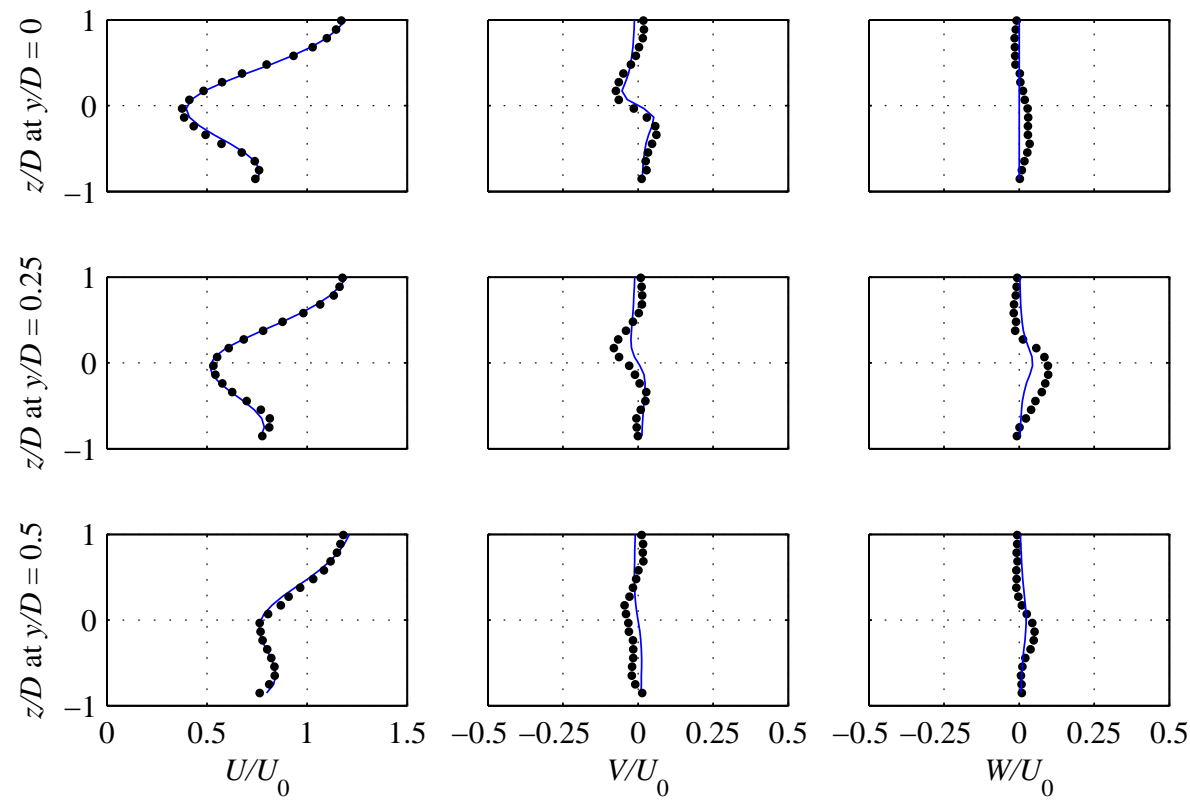

- Analytical Flow Field

- $\quad \mathrm{CFD}$

Figure 13: Comparison between the analytical flow field solution and the CFD results at different transverse offsets at $x / D=2$.

the slower tangential velocity components of the analytical solution show less agreement with the CFD. In most cases, the analytical solution underestimates the transverse and vertical velocity components of the wake, however the representative shape of the velocity profiles allows the analytical model to be a useful tool for exploring a range of VADCP orientation and location effects.

The RMS error of relative velocities between the CFD and the analytical flow model can be defined for the stream-wise velocity component by Equation 10, where $N=19$ for the bin locations within the range of $-1<z / D<1$.

$$
e_{U_{\mathrm{RMS}}}=100 \times \sqrt{\sum_{i=1}^{N}\left(\frac{U_{\mathrm{CFD}, j}-U_{\text {Analytical }, j}}{U_{0}}\right)^{2} / N} \%
$$

A summary of the errors between the CFD solutions and the analytical flow model are presented in Table 4.

A VADCP instrument is modelled in this analytical flow field simulation 


\begin{tabular}{cccc}
\hline & $y / D=0$ & $y / D=0.25$ & $y / D=0.50$ \\
\hline$e_{U_{\mathrm{RMS}}}(\%)$ & 1.99 & 2.75 & 2.11 \\
$e_{V_{\mathrm{RMS}}}(\%)$ & 2.14 & 2.79 & 2.63 \\
$e_{W_{\mathrm{RMS}}}(\%)$ & 1.97 & 3.42 & 1.67 \\
\hline
\end{tabular}

Table 4: Error analysis for the analytical model of the wake velocity field relative to the CFD solution

in the same manner as in the flow field generated using CFD. The analytical flow model does not include the recovery of the velocity deficit or the dissipation of the tangential velocity (swirl) in the wake of the turbine. This is considered to have a relatively minor effect on the results within the spatial resolution of the VADCP.

\subsection{Instrument Orientation Effects}

For homogeneous flow conditions, Mueller and Oberg [32] identify that, mathematically, rotating the instrument does not introduce bias in the transformed velocity measurement. However the orientation of the instrument becomes significant in non-homogeneous flow.

To explore the effect of instrument orientation, a VADCP was modeled in the analytical flow field solution to the CFD results at location $x / D=2$ directly downstream of the MHK device. The VADCP was rotated in yaw in $22.5^{\circ}$ increments. A comparison of the calculated and reference velocities is presented in Figure 14. When Beam 1 and Beam 2 are no longer aligned with the flow, measurement errors develop in the calculation of $U$ as well as $V$.

The sensitivity to the orientation of the instrument is shown in Figure 15. The error between the calculated VADCP result and the reference analytical velocity field is defined by Equation 7 . The solution was statistically stable when $t_{a}=180 \mathrm{~s}$.

The observed error is introduced in to the VADCP velocity calculations in two ways:

1. The $V$-velocity directly above the instrument is different to the $V$ velocity at the beam locations. This is more pronounced above the turbine hub height were the beams have diverged further.

2. The $W$-velocity component is non-homogeneous between beam pairs

In the default orientation $\left(\phi=0^{\circ}\right)$, the $U$-velocity in Beam 1 and Beam 2 are identical and equal to that directly above the instrument (ignoring the 

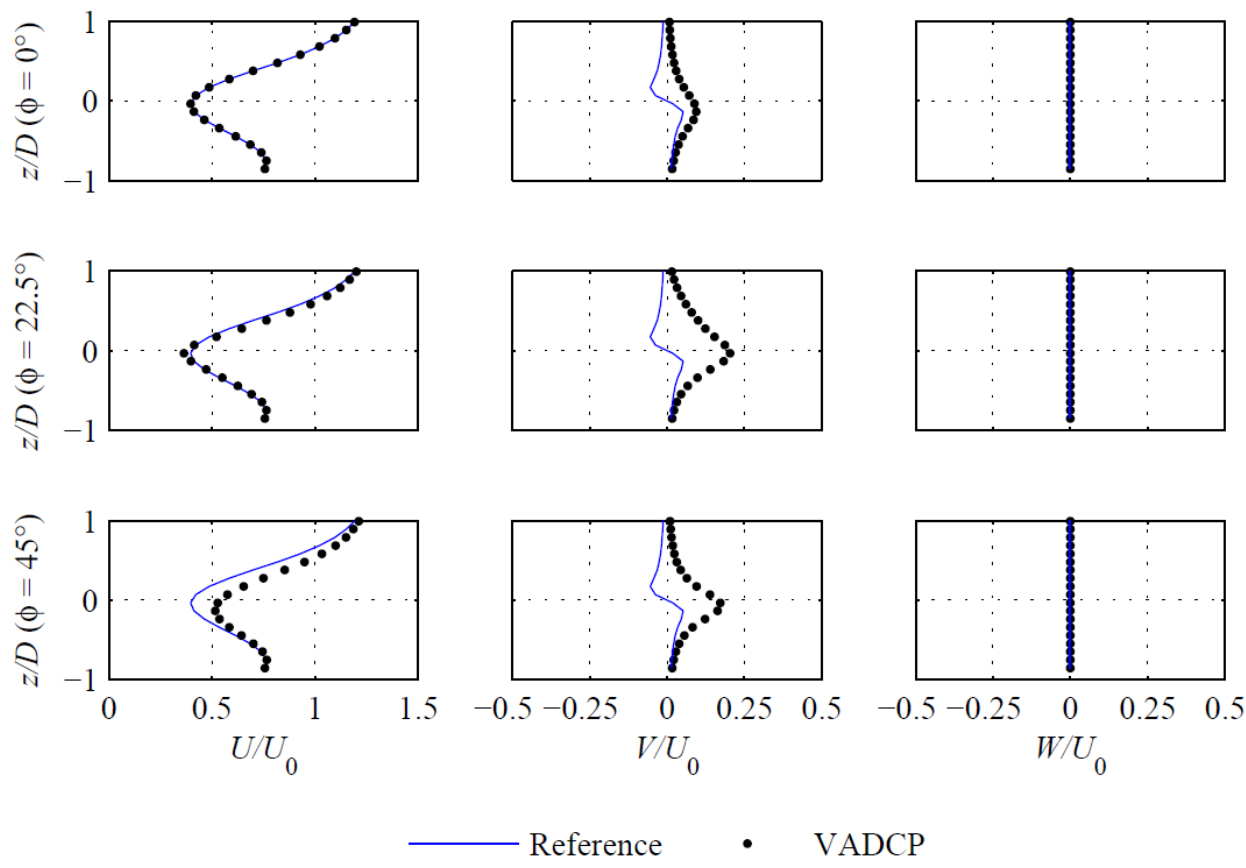

Figure 14: Comparison of calculated VADCP results and reference flow field velocities from the analytical solution to the velocity field at $x / D=2, y / D=0$.

wake recovery). The $U$-velocity is therefore quasi-homogeneous in the vertical plane at a particular vertical location. The $V$-velocity is perpendicular to these beams in this direction and is therefore undetected. The $W$-velocity component is zero in this plane. The instrument error is therefore zero.

As the instrument is rotated, the $V$-velocity and $W$-velocity components are resolved by Beam 1 and Beam 2. The $V$-velocity at the beam locations is less than that directly above the instrument. Also the $W$-velocity component is no longer zero and acts in an equal and opposite sense in each beam. This contribution is additive in the calculation of the $U$-velocity (Equation $4 \mathrm{a}$ ), and thus an offset is introduced by the $W$-velocity component. The maximum stream-wise error magnitude of $U_{e}=0.20 U_{0}$ occurs at a yaw angle of $\phi \approx 30^{\circ}$. This is shown in the left hand plot of Figure 15.

For $\phi=0^{\circ}$, Beam 3 and Beam 4 are in the vertical plane perpendicular to the mean flow direction. The beam-wise velocity in Beam 3 is equal and in the opposite direction to that in Beam 4 as it would be in homogeneous flow. However, again, the $V$-velocity magnitude at the bin locations are less 

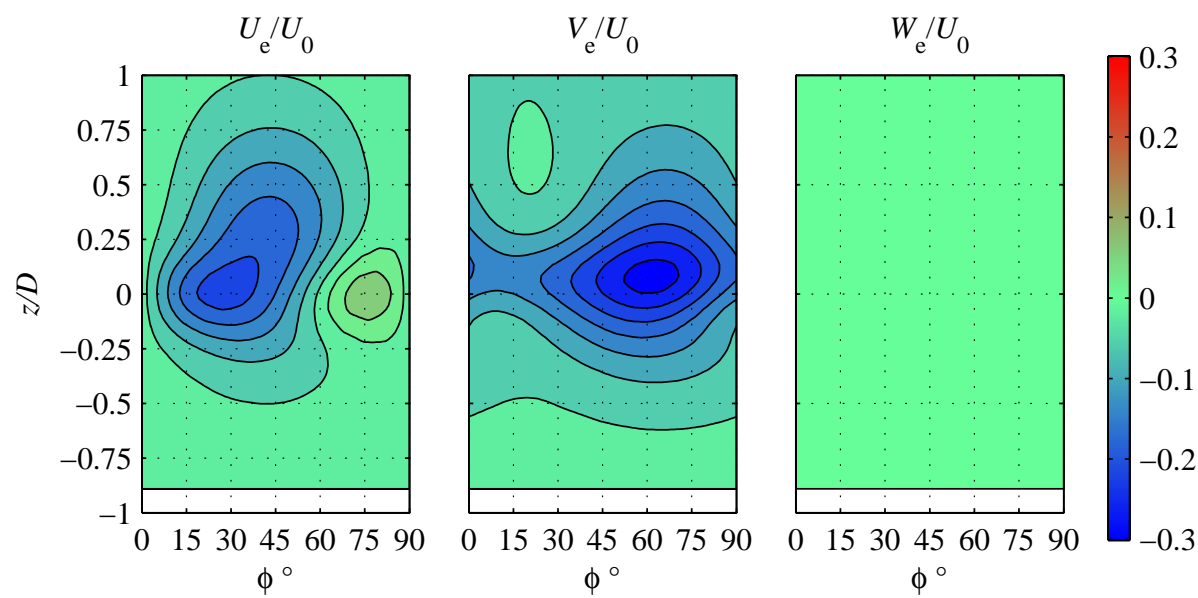

Figure 15: Sensitivity of VADCP results to instrument rotation at $x / D=2, y / D=0$.

than the $V$-velocity component directly above the instrument. Furthermore, the offset caused by the non-homogeneous vertical velocity component is present at zero yaw angle. The resulting measurement error of the VADCP is a maximum at an instrument rotation of $\phi \approx 60^{\circ}$, with the maximum transverse velocity error magnitude of $V_{e}=0.28 U_{0}$. This is shown in the central plot of Figure 15.

At the default orientation, the vertical velocity in the vertical plane aligned with the stream-wise flow direction is zero. Beam 1 and Beam 2 velocities are simply detecting the stream-wise flow velocity, and $b_{1}=-b_{2}$ as in homogeneous flow. Similarly, as indicated in Figure 9, the beam-wise velocity components of Beam 3 and Beam 4 have an equal and opposite value such that $b_{3}=-b_{4}$. This observation holds for all orientations of the device. Thus, in the calculation of the $W$-velocity component, the sum of the beam-wise velocities of both beam pairs is equal to zero, and this explains $W_{e}=0$ for all yaw angles at this instrument location.

\subsection{Transverse Location Effects}

The sensitivity to the transverse position of the instrument is shown by an analysis of the normalized measurement errors as a function of distance from the turbine centerline using the analytical solution to the velocity field in Figure 16.

The VADCP is able to resolve the stream-wise velocity component correctly for all transverse locations. This is because the flow is quasi-homogeneous 

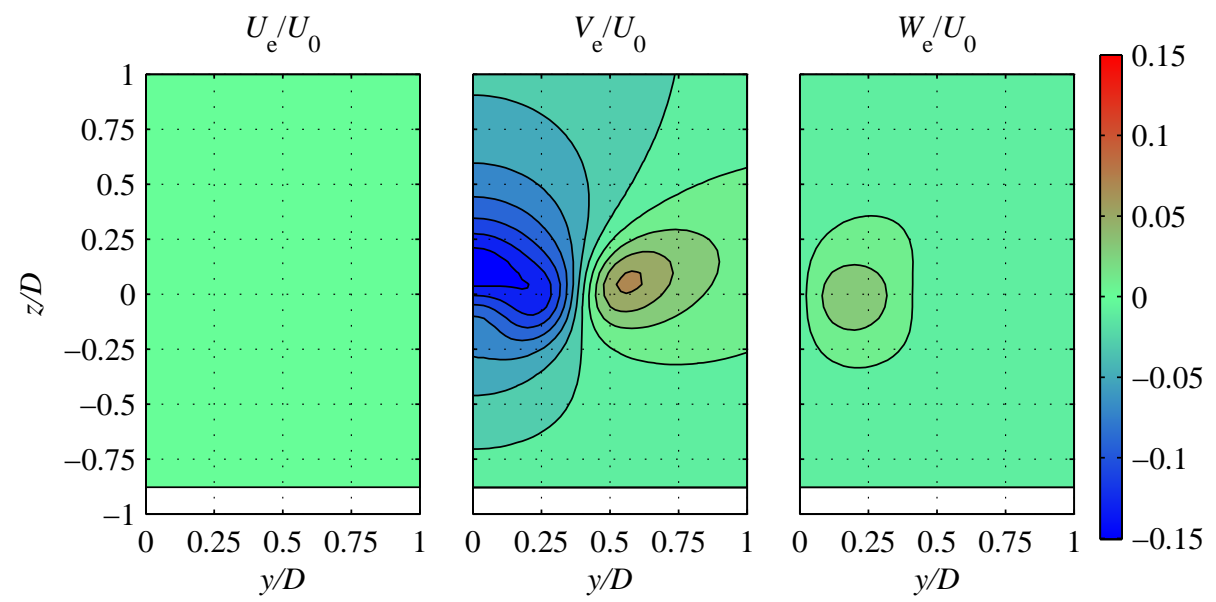

Figure 16: Sensitivity of VADCP results to transverse instrument position at $x / D=2$, $\phi=0^{\circ}$.

in the flow direction. That is, in the $x-z$ plane both the $U$-velocity and $W$ velocity components do not vary with distance behind the turbine (as the wake recovery and swirl dissipation are not modelled). As such, $b_{1}=-b_{2}$ when $\phi=0$ for all transverse instrument locations.

The maximum error magnitude in the transverse velocity component of $V_{e}=0.14 U_{0}$ occurs when the VADCP instrument is located directly behind the MHK device. As previously discussed, the error is the result of two factors. Firstly, the locations of the acoustic beams are not coincident with the reference measurement location directly above the instrument due to the beam divergence. Both the $V$-velocity and $W$-velocity which contribute to the beam velocity of Beam 3 and Beam 4 are different at the location of the acoustic beam to the nominal measurement location. Secondly, as the instrument is moved off-center, the beam-wise velocities are no longer of equal magnitudes, and the difference between the two beam velocities is increased. The local peak of $V_{e}=0.07 U_{0}$ at $y / D=0.58$ represents the transverse offset when Beam 3 intersects the maximum beam-wise velocity component of the swirl (right hand plot in Figure 17). At this offset Beam 4 is located outside of the region of significant swirl.

The error of the vertical velocity component is explained by the summation of the beam-wise velocities of all four beams in Equation 4c. An error is generated when the beam velocities of all four beams are not equal, as they would be in homogeneous flow. It is important to note that because the divergent beam angle is more closely aligned to vertical than horizontal 


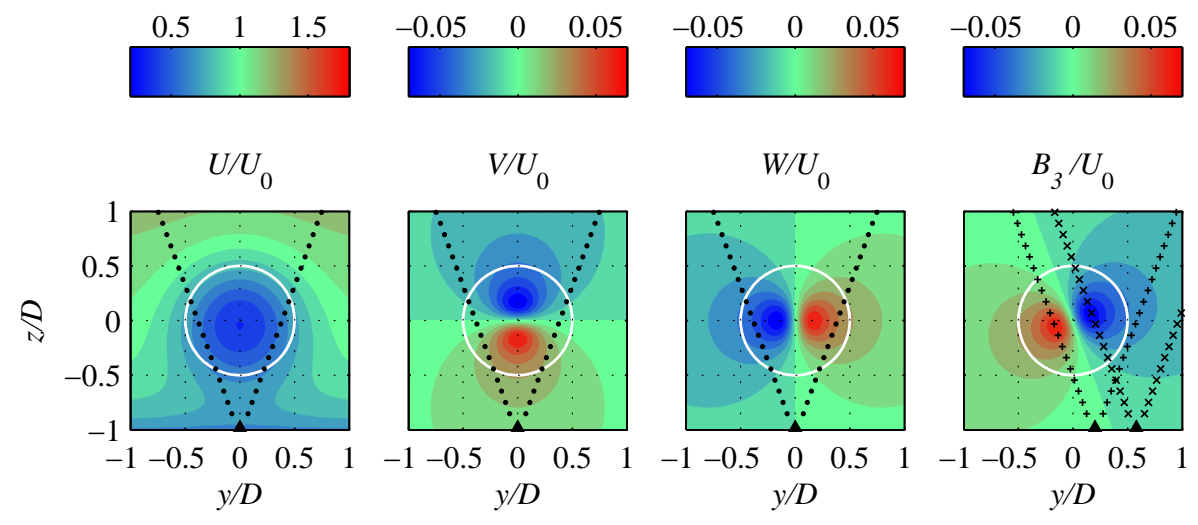

Figure 17: Normalized velocity components in the $y-z$ plane at $x / D=2$. Beam 3 (left) and Beam 4 (right) of the VADCP are shown for the case where the instrument is located at $y / D=0$ with dots, $y / D=0.20$ with plus symbols and $y / D=0.58$ with crosses. The turbine rotor diameter is indicated with a white line.

$\left(\theta=20^{\circ}\right)$, the vertical velocity component has the greatest influence on the beam-wise velocity.

The peak in the vertical velocity error occurs at the hub height when the instrument is located at $y / D=0.20$. At this position, Beam 1 and Beam 2 are located in the maximum vertical flow velocity, Beam 3 is close to the minimum vertical velocity in the swirl region Beam 4 is outside of the swirl region $(W=0)$. This case is shown in Figure 17 . The vertical velocity measured by the Beam 1 and Beam 2 pair is equal to the reference vertical velocity. However the combined contribution of the Beam 3 and Beam 4 pair is at its minimum. The result is the maximum error of $W_{e}=0.03 U_{0}$.

\section{Discussion}

One of the most common velocity measurements anticipated by an ADCP deployment in an MHK turbine wake is the stream-wise velocity component, which is aligned with the direction of the turbine axis. Both the available flow energy and the largest hydrodynamic forces on downstream arrays of devices are functions of this velocity component. From the VADCP analysis it follows that the theoretical instrument error is minimized when a beam pair is aligned with the flow direction (Figure 15). If this is achieved, then the velocity error is insensitive to lateral instrument location (Figure 16).

However Figure 15 demonstrates that misalignment from this orientation of $30^{\circ}$ can cause theoretical velocity errors exceeding $20 \%$ of the true 
stream-wise velocity at a location of $x / D=2$ and $y / D=0$. Such accurate instrument orientation is likely to require active control during deployment such as divers or remotely operated vehicles.

From this analysis the vertical velocity component remains relatively insensitive to instrument location and orientation. This mean velocity component is also typically most accurately resolved by real ADCP instruments, as the beams are more closely aligned with the vertical direction $\left(\theta<45^{\circ}\right)$.

In the configuration explored in this analysis, the additional beam allows a redundant measurement of the vertical velocity. It follows that comparing the vertical velocity calculated using the two beam pairs may provide information about the homogeneity of the instrument. Specifically, the vertical velocity can be calculated as shown in Equation 11.

$$
\begin{gathered}
\tilde{w}_{1}=\frac{b_{1}+b_{2}}{2 \cos \theta} \\
\tilde{w}_{2}=\frac{b_{3}+b_{4}}{2 \cos \theta}
\end{gathered}
$$

Locations and orientations exist where the two calculated vertical velocities $\left(W_{1}\right.$ and $\left.W_{2}\right)$ were very different, but the error between the VADCP velocity and the reference velocity was low. This method of comparing the independent measurements can indeed identify inhomogeneous flows, but does not detect whether such flows are problematic. This is best demonstrated by analyzing the coordinate transform presented in Equation 4c. The four beams velocities are summed in the numerator of the equation. Through this process, a wide range of beam-wise measurements are able to cancel or average out to the true velocity. Thus, while the flow may be quite non-homogeneous, the measurements are able to combine in such a way that zero error is observed.

Similarly, using the beam-wise velocity from all four available beams does not guarantee the maximum accuracy of the measurement. When one beam is located in a region where the velocity is notably different to the others, the measurement error would be reduced by not including that beam-wise velocity in a modification of the coordinate transform. It follows that a sensitivity analysis comparing the performance of VADCPs with three, four and five beams can also be achieved using the VADCP approach. These observations are of interest as the subject of future work using the VADCP model.

The velocity fields used in this analysis are intended to be realistic approximations to those which can be expected behind a full scale MHK device. 
The CFD model was validated against experiments in literature, and the analytical flow field was shown to represent the CFD results at $x / D=2$. For some full scale devices, this distance is of the order of $x=40 \mathrm{~m}$ from the device. The magnitudes of the errors identified using the VADCP should serve to raise awareness and provide recommendations for the appropriate use of diverging acoustic Doppler profilers to measure mean velocities in such non-homogeneous flows.

From comparison with published experimental results, it was seen that the DES approach used gave rise to higher rates of momentum mixing between the stream flow and the wake flow in comparison to actual field conditions. To remedy this overestimated mixing, other formulations such as large eddy simulation could be implemented at a greater computational cost.

\section{Conclusions}

The capabilities of an ADCP instrument in non-homogeneous flow in the near wake of a MHK device have been explored through the analysis of a virtual ADCP instrument. The velocity field behind an operating three-bladed horizontal axis MHK turbine was resolved using unsteady CFD simulations. The velocity at the locations of the VADCP measurement locations were extracted from the CFD simulation over a period of $180 \mathrm{~s}$. The time-averaged stream-wise velocity deficit and tangential swirl components of the near wake were then compared with available literature.

The velocity calculated from the beam-wise velocity coordinate transform was shown to deviate from a reference flow velocity directly above the instrument in the non-homogeneous wake conditions. The stream-wise velocity component ( $U$-velocity) was successfully resolved when the instrument was aligned with the flow. A relative error of $V_{e} / U_{0}=0.17$ was calculated at the hub height at a distance of $x / D=2$ due to the tangential velocity component of swirl in the near wake. The relative RMS error over the rotor plane was calculate to be less than $2 \%$ by a distance of $x / D \geq 2, x / D \geq 1$ and $x / D \geq 6$ for the $U_{e, \mathrm{RMS}}, V_{e, \mathrm{RMS}}$, and $W_{e, \mathrm{RMS}}$, respectively.

In order to efficiently perform a sensitivity analysis on the effect of the orientation and transverse location and rotation of the VADCP, an analytical flow velocity field was developed based on the time-averaged results of the CFD analysis. This analytical solution captured the shear boundary layer and velocity deficit in the stream-wise velocity as well as the swirl effects at $x / D=2$. For the default instrument orientation, A maximum error in the $V$-velocity occurs when the instrument is located directly behind the turbine, and a maximum $W$-velocity error is found at $y / D=0.20$. For 
an instrument directly behind the MHK device, the maximum error in the $U$-velocity and $V$-velocity occurs at a yaw angle of $\phi \approx 30^{\circ}$ and $\phi \approx 60^{\circ}$, respectively.

\section{Acknowledgements}

This research was supported by the U.S. Department of Energy, Energy Efficiency and Renewable Energy, Wind and Water Power Program.

Pacific Northwest National Laboratory (PNNL) is operated for the U.S. Department of Energy by Battelle under Contract No. DE-AC06-76RLO 1830 .

Computations described here were performed using the facilities of the PNNL institutional computing center (PIC).

\section{References}

\section{References}

[1] A. Lohrmann, B. Hackett, L. P. Rø ed, High Resolution Measurements of Turbulence, Velocity and Stress Using a Pulse-to-Pulse Coherent Sonar (1990).

[2] A. E. Gargett, Observing Turbulence with a Modified Acoustic Doppler Current Profiler, Journal of Atmospheric and Oceanic Technology 11 (1994) 1592-1610.

[3] RD Instruments, ADCP Coordinate Transformation: Formulas and Calculations, Tech. Rep. July (1998).

[4] M. Simpson, Discharge Measurements using a Broad-Band Acoustic Doppler Current Profiler, Tech. rep., USGS, Sacramento, CA (2001).

[5] A. J. Souza, The use of ADCPs to measure turbulence and SPM in shelf seas, in: 2nd International Conference \& Exhibition on Underwater Acoustic Measurements: Technologies \& Results, 2010.

[6] J. Thomson, B. Polagye, M. Richmond, V. Durgesh, Quantifying turbulence for tidal power applications, MTS/IEEE Seattle, OCEANS 2010 (4).

[7] V. S. Neary, B. Gunawan, C. Hill, L. P. Chamorro, Near and far field flow disturbances induced by model hydrokinetic turbine: ADV and ADP comparison, Renewable Energy 60 (2013) 1-6. 
[8] B. Gunawan, V. S. Neary, ORNL ADCP Post-Processing Guide and MATLAB Algorithms for MHK Site Flow and Turbulence Analysis, Tech. Rep. September (2011).

[9] E. A. Nystrom, C. R. Rehmann, K. A. Oberg, Evaluation of Mean Velocity and Turbulence Measurements with ADCPs, Journal of Hydraulic Engineering 133 (December) (2007) 1310-1318.

[10] J. A. Boldt, Use of Numerical Simulations to Investigate the Performance of a Virtual Acoustic Doppler Current Profiler in Characterizing Flow, Masters thesis, University of Illinois (2013).

[11] T. Tokyay, G. Constantinescu, J. A. Gonzalez-Castro, Investigation of Two Elemental Error Sources in Boat-Mounted Acoustic Doppler Current Profiler Measurements by Large Eddy Simulations, Journal of Hydraulic Engineering (November) (2009) 875-887.

[12] J. B. Richard, J. Thomson, B. Polagye, J. Bard, Method for identification of Doppler noise levels in turbulent flow measurements dedicated to tidal energy, International Journal of Marine Energy 3-4 (2013) 52-64.

[13] L. Myers, A. S. Bahaj, Near wake properties of horizontal axis marine current turbines (2009) 558-565.

[14] S. C. Tedds, I. Owen, R. J. Poole, Near-wake characteristics of a model horizontal axis tidal stream turbine, Renewable Energy 63 (2014) 222235.

[15] H. Shiu, C. P. van Dam, E. Johnson, M. Barone, R. Phillips, W. Straka, A. Fontaine, M. Jonson, A Design of a Hydrofoil Family for CurrentDriven Marine Hydrokinetic Turbines, in: ICONE20/POWER, Anaheim, CA, 2012.

[16] M. Barone, Preliminary Design of a Reference Marine Hydrokinetic Turbine, Tech. rep., Sandia National Laboratories, Albuquerque, NM (2010).

[17] A. A. Fontaine, W. A. Straka, R. S. Meyer, M. L. Jonson, A 1:8.7 scale water tunnel verification and validation test of an axial flow water turbine, Tech. Rep. August, Applied Research Laboratory, Pennsylvania State University (2013). 
[18] M. Richmond, C. Rakowski, W. Perkins, J. Serkowski, Simulating Collisions for Hydrokinetic Turbines. FY2010 Annual Progress Report (November).

[19] P. Romero-Gomez, M. Richmond, Discrete Element Modeling Of Blade - Strike Frequency and Survival of Fish Passing Through Hydrokinetic Turbines, in: 2nd Marine Energy Technology Symposium, Seattle, WA, 2014, pp. 1-11.

[20] CD-adapco, User Guide, STAR-CCM+ Version 8.02 (2013).

[21] J. Thomson, B. Polagye, V. Durgesh, M. C. Richmond, Measurements of turbulence at two tidal energy sites in Puget Sound, WA, IEEE Journal of Oceanic Engineering 37 (3) (2012) 363-374.

[22] N. Jarrin, S. Benhamadouche, D. Laurence, R. Prosser, A syntheticeddy-method for generating inflow conditions for large-eddy simulations, International Journal of Heat and Fluid Flow 27 (2006) 585-593.

[23] M. C. Richmond, P. D. J. Romero-Gomez, C. Rakowski, Simulating Collisions for Hydrokinetic Turbines, Tech. Rep. October, Pacific Northwest National Laboratory, Richland, WA (2013).

[24] P. Romero-Gomez, M. C. Richmond, Simulating blade-strike on fish passing through marine hydrokinetic turbines, Renewable Energy 71 (2014) 401-413.

[25] N. P. Dufresne, M. Wosnik, Velocity deficit and swirl in the turbulent wake of a wind turbine, Marine Technology Society 47 (4) (2013) 193205.

[26] S. R. Turnock, A. B. Phillips, J. Banks, R. Nicholls-Lee, Modelling tidal current turbine wakes using a coupled RANS-BEMT approach as a tool for analysing power capture of arrays of turbines, Ocean Engineering 38 (11-12) (2011) 1300-1307.

[27] F. Maganga, G. Germain, J. King, G. Pinon, E. Rivoalen, Experimental characterisation of flow effects on marine current turbine behaviour and on its wake properties, IET Renewable Power Generation 4 (May) (2010) 498.

[28] T. Stallard, R. Collings, T. Feng, J. Whelan, Interactions between tidal turbine wakes: experimental study of a group of three-bladed rotors., 
769 Philosophical Transactions of the Royal Society A: Mathematical, Phys-

$770 \quad$ ical and Engineering Sciences 371.

771 [29] S. Pope, Turbulent Flows, Cambridge University Press, 2000.

772 [30] R. Susan-Resiga, G. Dan Ciocan, I. Anton, F. Avellan, Analysis of the 773 Swirling Flow Downstream a Francis Turbine Runner, Journal of Fluids $774 \quad$ Engineering 128 (January) (2006) 177.

775

776

777

778

779

780

781

782

[31] Y. Hu, S. Parameswaran, J. Tan, S. Dharmarathne, N. Marathe, Z. Chen, R. Grife, A. Swift, Computing turbulent far-wake development behind a wind turbine with and without swirl, Wind and Structures 15 (2012) 17-26.

[32] D. S. Mueller, K. A. Oberg, Discussion of Near-Transducer Errors in ADCP Measurements: Experimental Findings by Marian Muste, Dongsu Kim, and Juan A. González-Castro, Journal of Hydraulic Engineering 137 (8) (2011) 863-866. 